\title{
ENTRE APATÍA Y COLABORACIÓN: ACTITUDES HACIA LAS ESCUELAS RURALES federales en la Región de San Bartolomé de Los Llanos, Chiapas, 1926-1940
}

\author{
Between Apathy and Collaboration: Attitudes about the Rural Federal Schools in the Region of San \\ Bartolomé de Los Llanos, Chiapas, 1926-1940
}

Óscar Javier Barrera-Aguilera

Resumen: Este estudio permite conocer las particularidades que revistió la implementación del proyecto de escuelas rurales federales -impulsado por la Secretaría de Educación Pública — en algunas de las poblaciones ubicadas en las terrazas en descenso hacia el valle central del río Grijalva en el estado de Chiapas. Con la finalidad de ilustrar las diferentes adaptaciones, contrastamos las experiencias que el proyecto afrontó en casos de comunidades tseltales, como Amatenango del Valle y Aguacatenango, y de poblaciones mestizas, como Teopisca, Tzimol y Socoltenango. Los casos analizados nos permiten preguntarnos por las alianzas y conflictos de intereses que surgieron entre las comunidades, los maestros y las autoridades a raíz de la implementación de este proyecto educativo.

Palabras clave: adaptaciones, alianzas, castellanización, conflictos, escuelas rurales, maestros.

Abstract: This study allows knowing the particularities that the implementation of the federal rural schools project — driven by the Secretaría de Educación Pública - had in some populations located in the descent towards the central valley of the Grijalva river terraces in Chiapas. In order to illustrate the different adaptations, we contrast the experiences that the project faced in cases of Tzeltal communities, as Amatenango del Valle and Aguacatenango, and mestizo populations, like Teopisca, Tzimol and Socoltenango. Analyzed cases allow us to ask about alliances and conflicts of interest that arose between the communities, teachers and the authorities as a result of the implementation of this educational project.

Keywords: adaptations, alliances, Castilianising, conflicts, rural schools, teachers.

Óscar Javier Barrera Aguilera, maestro en Historia por El Colegio de México. Temas de especialización: discursos y procesos de mestizaje en Colombia y Chiapas a lo largo del siglo XIX. Correo electrónico: ojbarrera@colmex.mx.
Enviado a dictamen: 02 de noviembre de 2012 Aprobación: 12 de diciembre de 2012 Revisiones: 1 


\section{Introducción}

De unos años para acá, la tesis del Estado como un Leviatán que se impuso sobre las regiones ha sido abandonada por gran parte de la historiografía interesada en el México posrevolucionario. Inicialmente se había sugerido que a lo largo de los años 1930 los maestros y las escuelas rurales conseguían reemplazar al cura y a la Iglesia, y que el gobierno federal lograba desplazar a los caciques de turno. Sin embargo, cada vez se hace más evidente que en la práctica el programa educativo de la Secretaría de Educación Pública tuvo que negociar con los habitantes y adaptarse a las condiciones particulares de cada localidad (Vaughan, 2000; Quintanilla y Vaughan, 1997).

Chiapas ha sido considerado como un laboratorio desafiante para el estudio de este programa por varias razones; entre ellas: su lejanía del centro del país y sus relaciones históricas con Guatemala; su diversidad étnica y geográfica; la pobreza y el carácter rural de la mayoría de su población, y la presencia de tradiciones de autonomía política (Lewis, 2005). Con el fin de contribuir a la comprensión de las particularidades regionales y subregionales de este estado, nos proponemos estudiar la experiencia de las escuelas rurales federales en algunas poblaciones de la región de San Bartolomé de Los Llanos, de las cuales unas eran indígenas -Amantenango del Valle y Aguacatenango-, en otras convivían indígenas y ladinos ${ }^{1}$ - Venustiano Carranza o Villa Las Rosas-y algunas eran definidas como mayoritariamente mestizas -Socoltenango, Teopisca o Tzimol- (Helbig, 1964; Viqueira, 1995 y 1997). En este escrito centramos la indagación en los casos de Teopisca, Amatenango del Valle, Aguacatenango, Tzimoly Socoltenango (vermapal).

\section{Las políticas educativas de la Secretaría de Educación Pública, 1920-1940}

Entre 1920 y 1940 la Secretaría de Educación Pública (SEP) se propuso incorporar los indígenas a la Nación mexicana a través de un proyecto educativo basado en su castellanización (Heath, 1972). De la mano de la antropología, los gobiernos de Álvaro Obregón y Plutarco Elías Calles buscaron regenerar moral y físicamente a la población mexicana con la finalidad de alcanzar la "homogeneidad racial" que requería el desarrollo armónico del país (Urías Horcasitas, 2002 y 2007).

En 1923, la Secretaría impulsó las Misiones Culturales bajo la premisa de conocer a las poblaciones indígenas para diseñar, de acuerdo con los resultados, la educación que más se adecuara a sus particularidades. La intención era que la Misión se convirtiera en un establecimiento a través de la introducción de aulas, talleres y parcelas, y del acompañamiento del maestro misionero por agrónomos, médicos y otros especialistas (Fell, 1989).

El 12 de marzo de ese mismo año, el maestro misionero Armando Guerra daba noticia de su arribo al municipio chiapaneco de Pinola - hoy Villa Las Rosas-. Tres días después, el maestro concluía que “algunas de las Escuelas Rurales de esta Zona están establecidas en lugares no apropiados por muchas circunstancias, una de las cuales es la de no estar en los centros indígenas y que concurren muy pocos alumnos." También aseguraba que, con sus rancherías El Plan y Canjob, el municipio reunía 4129 habitantes, cuya población indígena aportaba un —dudoso-95\% del total. ${ }^{3}$ En cualquier caso, hablantes de tseltal y practicantes del catolicismo, estos indígenas eran tan pobres que no habían podido levantar una escuela. Como en el resto del país, este proyecto fue abandonado debido a la falta de recursos y de constancia por parte del gobierno federal, y de confianza por parte de la comunidad.

Tras la experiencia de las Misiones Culturales, el posterior proyecto de la Casa del Estudiante Indígena fue un experimento del gobierno callista que pretendió reunir indígenas en la capital con la finalidad de enseñarles el idioma y los hábitos de los blancos para que posteriormente regresaran a compartirlos en su 
comunidad. Entre otras razones, el intento no rindió los frutos esperados porque los participantes - algunos de los cuales provenían de Chiapas - se rehusaron a retornar con sus hermanos (Loyo, 1996).

Más allá del experimento de la Casa del Estudiante Indígena, la presidencia de Plutarco Elías Calles quiso hacer de la educación, la irrigación y el crédito la base del desarrollo del campo mexicano. Moisés Sáenz fue el arquitecto del proyecto educativo callista, abanderando una escuela que integrara la población indígena y rural por medio de la castellanización y el trabajo en comunidad. A pesar de sus buenas intenciones, este programa no contó con los suficientes asistentes a las escuelas debido a la desconfianza de los padres de familia, a la necesidad de los brazos de los niños para trabajar en el campo y al difícil acceso desde lugares apartados (Arce Gurza, 1981). No en balde, tras encontrar desolación, pobreza y caciquismo en el Carapan de 1932, Sáenz consideró la estrategia de incorporación a través de la educación demasiado simplista y propuso la integración económica y social de las poblaciones indígenas (Sáenz, 1936).

Como en los años anteriores, durante el gobierno de Lázaro Cárdenas la educación socialista quiso proveer a los indígenas de tierras, agua, herramientas, mercados y créditos con la finalidad de incorporarlos a la economía y sociedad nacionales. En algunas zonas los maestros rurales consiguieron impulsar la reforma agraria, movilizar a los trabajadores y campesinos e introducir la ideología revolucionaria. Con todo, en 1935 el inspector de la SEP en Los Altos de Chiapas, Manuel Castellanos, señalaba que las buenas intenciones de los educadores en la región habían tropezado con el interés de los enganchadores contratistas - y de los gobiernos locales por mantener a los indígenas trabajando endeudados en las plantaciones de tierras bajas y alcoholizados en las cantinas de San Cristóbal de Las Casas. Adicionalmente, las propias comunidades indígenas desconfiaban de los profesores ladinos monolingües, quienes generalmente atacaban su cultura (Lewis, 2005; Lewis, 2006).
Estas breves consideraciones sobre la política educativa del gobierno federal entre 1923 y 1940 nos ofrecen un contexto amplio para emprender el análisis del rostro que revistió su aplicación en la región de San Bartolomé de Los Llanos, es decir, en un caso particular dentro de un estado de por sí considerado marginal.

\section{Teopisca: iel rechazo al gobierno federal por una élite ladina?}

Quizá la primera adaptación que debió asumir cualquier proyecto de escuela rural fue la de las instalaciones donde iba a funcionar. La escuela rural federal Valentín Gómez Farías, de la población mayoritariamente mestiza de Teopisca, inicialmente funcionó en el mismo edificio de la casa municipal. No teniendo espacio para las prácticas agrícolas, las autoridades del estado de Chiapas solicitaron la aprobación de la Secretaría de Gobernación para que el terreno anexo al templo fuera empleado con dicho fin. ${ }^{4}$ Una vez que abrió sus puertas, la escuela rural debió afrontar la escasa asistencia de los alumnos inscritos, que no pocas veces derivó en su completa deserción.

Al finalizar 1926, los logros en los terrenos agrícola y social parecían positivos. Los estudiantes practicaron la siembra y el cultivo de maíz y frijol y crearon una cooperativa para su explotación, con tan mala suerte que los amigos de lo ajeno se apropiaron de la cosecha del último. En vista de que 134 niños, distribuidos en cuatro grados, estaban siendo atendidos por sólo dos maestros, los padres no enviaban a sus hijos porque sabían que los educadores no podían atenderlos debidamente. A raíz de tales inconvenientes, el inspector Ramón Campillo hacía un par de recomendaciones: por una parte, pedía elevar el centro educativo a la categoría de escuela primaria elemental rural dado que los cerca de dos mil habitantes del pueblo de Teopisca ${ }^{5}$ laboraban en la agricultura y vivían y se sostenían de ella; por otra, sugería al ayuntamiento que, considerando la 
gran afluencia de estudiantes a la escuela, pagara un ayudante para las niñas y otro para los niños.

La intermediación del inspector Campillo dio prontos resultados, pues a mediados de 1927 los dos maestros ya contaban con un ayudante municipal. La institución ya contaba con la bandera nacional y dos mil metros cuadrados cultivados de gramíneas, leguminosas y frutas, además de un gallinero y una conejera. Los niños ya habían conformado varias asociaciones, las cuales sirvieron para la colecta y venta de productos que resolvieron algunas premuras económicas. Una de las debilidades de la institución seguía siendo la imposibilidad de constituir la Junta de Educación dado que, en una ocasión, los padres de familia no asistieron a la reunión citada porque estaban recibiendo la visita de otras autoridades del estado.

Transcurridos dos años, el inspector empezó a manifestar algunas incomodidades con respecto a la escuela de Teopisca. Ramón Campillo lo expresaba de la siguiente manera en uno de sus informes: "Para terminar quiero indicar a usted que a mi juicio, la comunidad de Teopisca no ha sabido responder a la ayuda que traté de impartirles en bien de la niñez y de las masas de campesinos." En su opinión, no se justificaba tener un profesor exclusivamente para los grados tercero y cuarto dada la inasistencia de los estudiantes. Al parecer, el problema era más complejo e involucraba las relaciones de poder al interior de la comunidad:

He omitido el aspecto políticosocial de la comunidad al que mucho se debe que la escuela no haya progresado cual es debido, ante las continuas discordias de cariz político que mantienen divididas a las familias y cuya trascendencia refluye hacia la escuela como factor disolvente o retardatario.?

A finales de agosto de 1927 se autorizó que la escuela ocupara la casa cural abandonada — es muy probable que esta iniciativa fuese favorecida por la persecución religiosa que se vivió en esos tiempos-. Sin embargo, a finales de 1930 el director de educación del estado denunciaba que las autoridades y algunos particulares de Teopisca estaban pidiendo que la escuela entregara los salones anexos al templo. En apoyo a su reclamo citaba las palabras del maestro de la escuela, Rosauro Navarro, quien acusaba a los aristócratas del pueblo de querer reducir el plantel "a la cocina o la calle". ${ }^{8} \mathrm{El}$ director de educación cuestionaba específicamente al agente de correos, quien a la vez fungía como mayordomo del templo de San Agustín y junto con los clérigos estorbaba la asistencia de los alumnos a la escuela, negaba el progreso alcanzado por la misma y gestionaba el desalojo de los anexos del templo.

Tras el entusiasmo de los primeros años, la visita de inicios de 1931 recalcaba que en la escuela rural federal de Teopisca no habían sido fomentadas ni las industrias locales ni la cría de animales ni los cultivos, así como tampoco había ya una cooperativa de niños o de adultos. A mediados del mismo año, la institución continuaba sin material escolar, agrícola e industrial, carecía de superficie cultivada y volvía a funcionar en el edificio del ayuntamiento. Lo más sobresaliente era la inasistencia, de la cual se responsabilizaba al presidente municipal, quien al parecer se hacía de la vista gorda en su tarea de exigir la enseñanza obligatoria.

Si bien se señalaba que la "raza" predominante en la villa de Teopisca era la mestiza y que todos los alumnos hablaban español, el inspector aclaraba que la mayor parte de los mismos provenía del barrio denominado Pueblo Viejo, el cual era prácticamente indígena en su totalidad. No es de extrañar, entonces, que entre las labores desarrolladas por el maestro se encontrara la de enseñar a los estudiantes a dormir en camas. A pesar de tal iniciativa "civilizatoria", el informe de septiembre de 1931 concluía enfatizando el poco acercamiento conseguido entre la escuela y la comunidad, el cual se veía reflejado en el escaso número de celebraciones cívicas y en la precaria asimilación de conocimientos concernientes a la vida de los más grandes mexicanos y a la organización política del municipio y la Federación. 
A mediados de 1933, el desempeño de la escuela seguía cayendo en picada. Un informe de puño y letra del director de la escuela denunciaba la tala indiscriminada de árboles y la desatención mostrada por la comunidad a sus consejos. Las autoridades federales habían abandonado la institución a su propia suerte pues ya llevaba un buen rato sin recibir visita del inspector. Nuevamente, las reiteradas divisiones políticas habían impedido la formación de cooperativas y el presidente municipal no daba cumplimiento a la Ley de Educación en el sentido de que no fomentaba la asistencia a la escuela. Adicionalmente, el maestro denunciaba que las señoritas Molinares y la Sra. Mercedes Zúñiga de Castro estaban ofreciendo clases particulares en sus hogares, hecho que contribuía a menguar el de por sí mínimo número de asistentes a la escuela.

Hasta donde fue posible seguir la huella de la escuela rural federal de Teopisca encontramos estos problemas ya recurrentes. El ayuntamiento continuó haciendo lo posible por interrumpir las actividades del plantel y la inasistencia de los alumnos fue adjudicada a la existencia de "restos de reaccionarismo y fanatismo" en la comunidad, los cuales se referían a la institución de manera poco edificante.

En la oposición de las autoridades estatales y municipales, de los grupos acomodados y de los clérigos al uso del espacio parroquial por parte de la escuela, en el escaso aliento proveniente del presidente municipal a cualquier actividad propuesta por la misma, y en la preferencia por la enseñanza impartida por particulares, podemos percibir la incomodidad que, por entonces, provocaba en ciertos grupos de Teopisca la entrada del maestro rural, quien en últimas era visto como un intruso que representaba al Estado y venía a desacomodar el orden social. No tenemos los suficientes datos para asegurarlo, pero es probable que en este caso las autoridades locales y algunas de las familias más influyentes hayan triunfado en defender las prerrogativas alcanzadas en años anteriores sobre la reciente iniciativa federal de hacer presencia en la comunidad a través de la educación.

El escaso conocimiento de temas cívicos e históricos bien podría reflejar el poco éxito de la escuela en su intención de integrar a la población en la sociedad nacional. Teniendo en cuenta el origen de la mayoría de los estudiantes recibidos, cabe preguntarse si lo que nos está revelando la escuela rural federal de Teopisca es la oposición de las élites ladinas a la incursión de un gobierno federal como protector de la población indígena.

\section{Amatenango del Valle: zuna comunidad tseltal colaboradora?}

La escuela rural federal de Amatenango del Valle estaba ubicada a 36 kilómetros de San Cristóbal de Las Casas y a cuatro de Teopisca. A diferencia de lo acontecido en esta última población vecina, aquí la institución atendió los requerimientos de una localidad predominantemente tseltal. De acuerdo con el inspector Ramón Campillo, sus habitantes vivían avecinados en parajes y se dedicaban a la agricultura y la cerámica, siendo los decorados de esta última "groseros y rudimentarios". En su visita de julio de 1927, el inspector señalaba que: "Todos hablan el idioma español, además de su lengua nativa. No obstante esto y la proximidad que guardan con el pueblo de Teopisca, habitada esta última (sic) en su mayor parte por mestizos, en nada han modificado sus costumbres ancestrales". ${ }^{9}$

Desde los primeros años el maestro consiguió impulsar la fabricación de esteras y la formación del Comité de Educación. Como sucedió en la mayor parte de la región, desde muy temprano la Federación estuvo reconviniendo al presidente municipal para que cumpliera con sus deberes dotando la escuela, entregando el convento del lugar — tal vez vacío a causa de la persecución religiosa - con el fin de establecerla allí y fomentando la asistencia de los estudiantes y la creación de cooperativas. 
Las condiciones del área donde se hallaba ubicada la escuela no eran nada buenas. El terreno pedregoso impedía la agricultura, presentándose la necesidad de buscar un lugar fértil y con agua para reubicar las instalaciones. Tras un año de haber entrado en funcionamiento, el plantel no contaba siquiera con bandera ni mucho menos había impulsado el trabajo con animales. Incluso la sección nocturna para adultos era vista con el mayor desprecio y la labor educativa se vio entorpecida a causa de que la comunidad sufría una epidemia de tifo, la que, por cierto, el inspector Campillo adjudicaba a la pobreza, al atraso y a la suciedad en que vivía el pueblo de Amatenango. Teniendo en cuenta lo anterior, no es de extrañar que el año escolar de 1927 haya terminado con una inasistencia superior al 50\%, pues de cincuenta inscritos sólo terminaron el ciclo 21.

Tal era el estado de cosas en Amatenango que dos años después las pocas mejoras en el campo de cultivo, el jardín y el gallinero les merecieron una felicitación a los maestros Jesús Castillo y Catalina Calvo por parte del inspector Campillo. Lo que más inquietaba al inspector era que, tras cinco años de estar funcionando la escuela, ningún niño conocía la organización política de la Federación a la que pertenecía ni cómo estaba estructurado el municipio o cuál era su forma de gobierno.

Aunque el anterior estado de cosas era poco alentador, a partir de 1932 podemos observar algunos cambios en la escuela rural federal de Amatenango. Ahora los niños podían dar cuenta de la vida de los más grandes mexicanos y de la forma en que estaban organizados el municipio y la entidad federativa. Seguramente los avances se debían al buen entendimiento entre la escuela y la comunidad, lo cual también había conducido a impulsar un club de agricultura que —en palabras del inspector - serviría de ejemplo para otras poblaciones. A inicios de 1933, el plantel ya contaba con biblioteca, oficina de correos, línea telefónica, vitrola y hasta museo. Adicionalmente, se había conformado una cooperativa de alfarería para que las niñas pudieran vender los productos que elaboraban. El único lunar era que el aseo personal aún dejaba mucho que desear.

Las principales características del pueblo eran resumidas del siguiente modo en 1934:

Amatenango es una congregación indígena, de la misma raza de los Chamulas, pero cuyas costumbres difieren un poco; hablan el mismo idioma Tzotzil (sic) pero con ligeras diferencias. Sin embargo, hay muchos que hablan el castellano. Su forma de gobierno es la misma de los Chamulas, resolviendo los asuntos más delicados un Consejo de Ancianos que en mayor parte frisan el siglo, en tanto que los Gobernadores de Parajes son elementos jóvenes de relativa cultura por haber servido de mocitos en familias de esta ciudad (San Cristóbal). ${ }^{10}$

Esta descripción revela que las autoridades educativas federales empleaban el estereotipo de los chamulas los cuales son tsotsiles - para describir a poblaciones tseltales como la de Amatenango del Valle. Este factor nos muestra el desconocimiento - o desinterés- de tales autoridades hacia las diferencias lingüísticas y las conexiones socioeconómicas entre las poblaciones de Los Altos de Chiapas y la región de San Bartolomé de Los Llanos. En cualquier caso, el uso de estereotipos ratifica el objetivo del programa de escuelas rurales federales: integrar a los indígenas en la sociedad mexicana sin importar sus particularidades.

Las autoridades federales se sentían orgullosas de haber convertido a los indígenas de Amatenango del Valle en entusiastas de la escuela, quienes incluso pusieron a disposición del profesor Gaudencio Dávila los libros y útiles del plantel educativo que habían empleado con anterioridad - tal vez una iniciativa de origen puramente local-. Curiosamente, también advertían que estos indígenas ahora tenían una idea más clara de la religión católica, pues aun cuando sus supersticiones habían aumentado, el culto a los astros había quedado en el olvido. 
No podemos lanzar una conclusión certera acerca de la experiencia de la escuela rural en Amatenango del Valle durante estos años. A pesar del optimismo de las autoridades educativas por los logros de la comunidad en el camino a la integración nacional, el corto período de tiempo que abarca el expediente (1926-1934) y el hecho de que en la actualidad el pueblo siga siendo principalmente tseltal nos hacen dudar de tales alcances. En otras palabras, una cosa es memorizar autoridades y prohombres de la patria y otra muy distinta abandonar prácticas y creencias asociadas a una configuración político-económica que mantenía a esta población indígena bajo el dominio de los ladinos de San Cristóbal de Las Casas.

\section{Aguacatenango: \\ ¿la apatía de una población tseltal?}

Descendiendo hacia el oriente 15 kilómetros por el valle que empieza en Teopisca se llega a la comunidad de Aguacatenango, que a su vez dista 53 kilómetros de la ciudad de San Cristóbal. A inicios de los años 1930 contaba con cerca de 600 habitantes, en su totalidad indígenas, de los cuales casi todos los hombres sabían el castellano, mientras que mujeres y niños se comunicaban principalmente en tseltal. Un informe de la primera mitad de los años treinta resumía su situación señalando que, a pesar de que eran "refractarios a la civilización", sus pobladores se habían habituado a tener escuela."

Durante los años veinte, la preparación de ixtle, la siembra de maíz y la hechura de sombreros de palma fueron las principales actividades productivas de la población de Aguacatenango. ${ }^{12}$ A principios de la década de los treinta, la situación precaria en que se encontraba la escuela no había cambiado para nada. Con sabor amargo, el inspector Epigmenio de León aplaudía al maestro Jesús Castillo por la iniciativa de desarrollar "juegos organizados en la plazuela del lugar, con el fin de atraer a los niños indígenas y algunas niñas para encariñarlas con la escuela; pero [que] por excesivo celo de los padres y por la poca costumbre de tener maestro, no ha sido posible hacer concurrir."13 De la misma manera, el impacto de la escuela en la promoción de actividades cooperativas y en la enseñanza de la historia y la organización política había sido mínimo.

En octubre de 1930, las autoridades municipales trataron de limpiar la mala imagen que el inspector tenía de su gestión señalando que, en cuanto al mejoramiento de la infraestructura de la localidad, el alcance de la escuela había sido mayor gracias a la acción simultánea del maestro, la comunidad y la agencia municipal. Dichas autoridades aplaudían la labor del maestro rural Damián Chávez, no sólo por haber promovido - después de dieciséis años de abandono - la rehabilitación de la casa escolar, sino por haber hecho llegar el agua potable a la plaza y haber velado por el mejoramiento de las calles del pueblo. El gobierno local también celebraba la colaboración de la comunidad ayudando a levantar la casa de la escuela ante la falta de fondos públicos. En pocas palabras, la agencia municipal resaltaba la forma en que el profesor había dirigido a la comunidad y le suplicaba que jamás se negara a fomentar la civilización, "la única manera en que se salvaría la patria." ${ }^{14}$

Con la información recabada es imposible establecer claramente las alianzas entre las personas mencionadas y la veracidad de los logros enunciados. No obstante, apenas habían transcurrido seis meses desde que las autoridades municipales elogiaron su gestión, cuando el inspector José Inés Estrada informaba que el maestro recientemente cesado se había llevado con él las gallinas y las palomas pertenecientes a la escuela. Entre las peticiones hechas en ese entonces por el inspector a las autoridades municipales resaltan: que exigieran a los padres la asistencia de sus hijos; que gestionaran junto a los vecinos la dotación de muebles y la explotación del área de cultivo; que fomentaran las actividades 
deportivas y teatrales para despertar entusiasmo en la comunidad; y que impulsaran inmediatamente, y antes que todo, la confección de la bandera de la escuela. ${ }^{15}$

En el informe preparado sobre la visita de septiembre de 1931, el inspector Estrada fue enfático en señalar que la comunidad se mostraba absolutamente renuente a todos los trabajos provenientes de la escuela. Le preocupaba que hasta la fecha sólo se hubieran inscrito niños y ninguna niña, y que - como sucedía en la mayoría de las escuelas de la región de San Bartolomé de Los Llanos - a la jornada nocturna únicamente asistían hombres.

El año siguiente, en vista de lo atrasados que estaban los cursos, se propuso redoblar el trabajo docente e impulsar el club de exploradores y las actividades deportivas. A pesar de que la escuela consiguió organizar varias agrupaciones, los niños terminaron 1932 sin saber nada de historia ni de organización política. En síntesis, la indolencia del maestro mantenía inactiva la institución.

En junio de 1933, Séptimo Pérez Palacios, director de Educación Federal, propuso que la plaza del maestro fuera aprovechada por la ranchería El Puerto - una población cercana cultural y geográficamente a Aguacatenango-, lo cual fue ratificado en el mismo mes por el entonces jefe del Departamento Rural, Rafael Ramírez. Así terminaba la historia de una escuela caracterizada por la escasa asistencia de alumnos y la poca colaboración del vecindario y las autoridades, factores que terminaron justificando su clausura.

Al parecer, en esta oportunidad los maestros estuvieron al servicio de las obras públicas impulsadas por las autoridades municipales, quienes desalentaron las actividades puramente escolares. Probablemente la comunidad indígena, con arraigadas costumbres como su lengua y la restricción a las mujeres de realizar actividades diferentes al hogar y la siembra—, también fue manipulada por los grupos dominantes locales para oponerse al proyecto educativo federal.

\section{Tzimol: zuna escuela modelo?}

Finalizando los años veinte del siglo pasado, Tzimol era definida como una población de ladinos dedicados al cultivo de la caña de azúcar y a la fabricación de panela. A diferencia de los casos de Teopisca, Amatenango del Valle y Aguacatenango, la escuela rural federal de Tzimol ofrecía condiciones físicas relativamente apropiadas. A pesar de las buenas intenciones, el dolor de cabeza inicial fue la ausencia de niñas y mujeres inscritas. $^{16}$

El primero de agosto de 1929 se reintegró el Comité de Educación con ayuda del inspector de la segunda zona escolar de Chiapas, el profesor Prefecto S. Rodríguez, quien explicó a los padres de familia las formas de nombramiento y las finalidades del mismo. Al inicio del siguiente año, el inspector impulsó reuniones sabatinas y dominicales para discutir acerca de la previsión, el antialcoholismo y el nacionalismo. La intención era que el vecindario, la autoridad y el maestro pugnaran por la implementación de los anexos más viables para imprimir a la escuela el tipo moderno que debía tener: gallinero, conejera, palomar, aviario, porqueriza y excusado. En aras de impulsar ese carácter innovador del plantel, debía fomentarse la asistencia, la puntualidad y la formalidad, al igual que las actividades prolengua y procálculo y los comités de limpieza y exploradores. ${ }^{17}$

Se produjo una calamidad que marcó los derroteros de la escuela a partir de la segunda mitad de 1932. El 17 de septiembre un fuerte aluvión inundó la población de Tzimol y perjudicó seriamente muchos edificios. Este hecho obligó al traslado y reconstrucción de la escuela con la ayuda de alumnos y profesores de todo el estado de Chiapas, quienes además contribuyeron a recolectar 4.25 pesos. Pasado casi un año de haberse presentado la inundación, y junto a campañas proárbol, prohigiene, proinfancia y promedidas del sistema métrico decimal moderno, el inspector José Estrada impulsó el desvío de las corrientes de agua que atravesaban la localidad, 
pues constituían una constante amenaza. Esta última medida fue complementada con la formación de una caja de ahorros ideada para enfrentar futuras eventualidades.

Superados los estragos provocados por las inclemencias del tiempo, en la segunda mitad de 1933 la escuela empezó a experimentar una notable mejoría en sus condiciones. Aunque carecían de impresos relacionados con la agricultura e industrias afines, los niños habían incorporado los principios de la cívica y la historia, y contaban con útiles y muebles completos. También podemos observar una intensificación de las actividades culturales y sociales promovidas por la escuela. En 1937, Fernando Utrilla — director encargado temporalmente de la escuela - ya era reconocido en todo Chiapas como un activo promotor. El informe que presentó sobre la visita de sus alumnos al festival organizado por la escuela rural El Puente - ubicada a seis kilómetros de Tzimol- es bastante ilustrativo:

Se reunieron como 300 campesinos, muchos de ellos nunca habían presenciado una sencilla fiesta. Para el desarrollo de estos festivales, llevo de esta Escuela una lámpara de gasolina que me es muy útil y que gusta mucho a los campesinos, guitarra y mandolina y el cuadro de Títeres [...] (ver foto l).

Yo tuve la satisfacción de hacerme entender por medio de los títeres a los campesinos, muchos de los cuales no habían querido enviar a sus hijos a la escuela ni tener ningún contacto con ella. El resultado fue; según comunica el Maestro de ahí, que la asistencia ha aumentado considerablemente y todos los campesinos se van acercando más al maestro en sus problemas económicos y sociales. ${ }^{18}$

El éxito de las actividades sociales y culturales en el avivamiento del compromiso comunitario con la escuela abandonó Tzimol con el maestro Fernando Utrilla. En 1942, fuera del excusado, la escuela carecía de anexos y la asistencia era sumamente deficiente. En pocas palabras, la marcha defectuosa del plantel respondía a que el maestro Rubén Pasquett —en su retorno a la dirección - no había podido adelantar ninguna labor social por la indiferencia de los padres de familia. No deja de ser curioso el decaimiento de una escuela que ante las incertidumbres de la naturaleza había respondido con ahorro y previsión, y que a través del arte y la cultura había conseguido acercarse a la comunidad. ¿Hasta qué punto este declive se debió al cambio de director, a la pérdida de interés de la comunidad o a las nuevas directrices en el gobierno federal con la presidencia de Manuel Ávila Camacho? Ésta es una pregunta que no podemos responder por ahora.

\section{Socoltenango: zuna comunidad politizada?}

En 1926 la escuela rural federal de Socoltenango entró a fomentar la fabricación de sombreros de palma por parte de una población mestiza y hablante de castellano. Funcionando en una pieza del cabildo carente de todo mobiliario, la escuela en realidad estaba dividida en una sección destinada a los niños y otra a las niñas, además de las jornadas nocturnas. Al año siguiente, la Junta de Educación confesó que no había hecho nada por la escuela de niños debido a su desacuerdo con Salvador Guillén —el director de la misma-, quien al parecer se ausentaba de sus labores para dedicarse a asuntos personales. La escuela de niñas, por su parte, estaba bajo la dirección de Lesbia Nájera y se encontraba en peores condiciones físicas que el plantel de niños. ${ }^{19}$

El año 1929 mostró una notable participación de la comunidad asociada con las escuelas. El 19 de junio fueron instalados el comité de mejoras y el de educación, los cuales en noviembre ya eran plenamente utilizados por los pobladores para velar por óptimas condiciones en la labor educativa. Los padres de familia solicitaban la verdadera formación de las escuelas, quejándose de que sus súplicas habían sido desatendidas desde hacía varios años por la Dirección General de Educación. Resulta paradójico que, después de haber cuestionado su comportamiento, la comunidad señalara que "la 
desmoralización" inició cuando el prefecto Salvador Guillén hizo entrega de la dirección de la escuela a la maestra rural Matilde Avendaño, a quien culpaban de haberse olvidado completamente de la labor social. Para colmo de males, la maestra vivía en completa riña con todos los habitantes de la comunidad. Por esta razón, los padres de familia se habían visto en la necesidad de retirar a sus hijos de la escuela antes de que ella los cosiera, como las recurrentes quejas señalaban que ya había hecho con algunos. ${ }^{20}$

Además de haber dejado pasar las fiestas patrias sin pena ni gloria, la maestra fue acusada de haber maquillado las estadísticas para hacer aparecer un número considerable de alumnos. De acuerdo con los denunciantes, el promedio mensual de alumnos asistentes a lo largo del año realmente no había pasado de 15, pero la maestra Avendaño se había valido de artilugios para hacerlo parecer mayor; señalaban que incluso había llevado a sus hijas, quienes estudiaban en Comitán, para hacerlas pasar por estudiantes suyas durante una visita realizada por el entonces inspector, Salvador Guillén.

Como en su visita el inspector Guillén impartió algunas clases, los habitantes asociaron como consecuencia de dicha labor el aumento en el número de alumnos asistentes a la escuela y el despertar del interés entre las niñas y los padres de familia. Esa experiencia llevó al Comité de Educación a concluir que los niños ya no querían una maestra sino un maestro, por lo que propusieron que les reasignaran a Salvador Guillén como profesor.

A partir de 1931 empezó a figurar una sola escuela para niñas y niños, ahora a cargo del maestro Fortunato Argueta, quien tuvo que trabajar a doble jornada por falta de ayudantes. Pareciera que, en aquel entonces, tuvo que reiniciar la escuela desde cero, pues en los años siguientes fueron recurrentes los llamados del inspector para que se organizaran agrupaciones de diferente índole. Igualmente repetidas fueron las solicitudes de habilitación de la casa cural para labores educativas dado el crecido número de estudiantes y las peticiones de ayuda a la autoridad y los padres de familia para que dotaran la escuela y tuvieran mayor contacto con ella.

Al parecer, a finales de los años treinta el interés de los pobladores de Socoltenango por las actividades de la escuela era notable. Puede percibirse en la siguiente petición que hacían al director de Educación Federal, identificándose ahora como miembros del Comité Ejecutivo Agrario y del Comité Municipal del Partido de la Revolución Mexicana:

Hoy venimos notando el abandono en que se encuentra la Escuela de este Pueblo. El maestro que se encuentra al frente de la misma nomás toca la campana y se va a su casa. [...] Llega unos 5 o 20 minutos para dar las clases y luego cierra la escuela. Por lo que ocurrimos a usted pidiendo una maestra que es nativa de este pueblo, que es una joven de espíritu netamente revolucionaria y social. ${ }^{21}$

Los vecinos aludían a María Cancino, quien en vacaciones había organizado veladas bailables y juegos deportivos en Socoltenango. La petición de nombramiento de la Srta. Cancino como maestra no fue un capricho del momento pues volvió a presentarse cuando Fortunato Argueta fue trasladado a Villa Las Rosas.

Es posible palpar en Socoltenango la aparición de algunos principios de lo que la historiografía ha denominado como la escuela socialista (Arce, 1981). El plan de trabajo educativo formulado para 1939 abarcaba programas de higiene, salubridad, mejoramiento económico, promoción social, construcción y recreación que partían de la escuela para extenderse a la comunidad. Por ejemplo, la propuesta incluía la construcción de ocho kilómetros de carretera y la formación del Club Femenil de Mujeres Revolucionarias.

Alrededor de la escuela rural federal de Socoltenango tenemos una activa comunidad que denuncia la negligencia de los maestros y de las autoridades 
municipales. ¿Cuál es el origen del papel activo de esta comunidad? No podemos establecer con certeza si la iniciativa política de los vecinos de Socoltenango se debe a la penetración concreta del proyecto educativo de la SEP, a la presencia del Partido de la Revolución Mexicana (PRM), a ambos factores o a otros de tipo diferente. Lo cierto es que la comunidad empleó el discurso revolucionario con la finalidad de presionar para que se nombrara personal docente más eficiente y cercano a sus aspiraciones.

\section{Reflexiones finales}

A ojos de sus intelectuales, los experimentos educativos rurales de la SEP durante las dos décadas que siguieron a 1920 arrojaban un par de conclusiones inmediatas. En primer lugar, que la condición subordinada de los indígenas en la sociedad no se debía a factores raciales sino materiales y sociales. En segunda instancia, tanto los congresos indígenas regionales de 1939 como la Conferencia Indigenista Interamericana, celebrada en Pátzcuaro al año siguiente, reconocieron el derecho de los indígenas a preservar su lenguaje y costumbres, haciendo un llamado a favor de las escuelas bilingües (Lewis, 2006).

Recientemente Stephen Lewis ha propuesto que los proyectos adelantados por la SEP entre 1920 y 1940 fueron aceptados por poblaciones indígenas que ya venían incorporándose a la sociedad mexicana, mientras que en Los Altos de Chiapas fueron un fracaso debido a las condiciones históricas de marginamiento y explotación (Lewis, 2005). ¿Qué podemos decir al respecto a partir de los casos que estudiamos para la región de San Bartolomé de Los Llanos?

Es muy probable considerar lo que nos presenta el caso de la escuela de Teopisca como la oposición de una mayoría ladina a la incursión de un Estado federal con una política favorable a la minoría indígena. Así, podemos advertir la importancia de estudiar poblaciones mestizas y multiétnicas, en las cuales las diferencias de intereses y las relaciones desiguales entre sus pobladores ofrecieron variadas respuestas al proyecto educativo posrevolucionario.

De acuerdo con lo expresado por las autoridades educativas federales, podríamos concluir que la población tseltal de Amatenango del Valle recibió positivamente el proyecto escolar. Visto desde el presente, podríamos decir que en el largo plazo el proyecto no consiguió la integración de esta población indígena a la sociedad mexicana. Sin embargo, debemos cuidarnos de simplificar el análisis del proyecto de las escuelas federales en términos de su éxito o fracaso en la incorporación de las poblaciones rurales. Queda para una próxima investigación determinar en qué medida la experiencia de las escuelas rurales contribuyó a afirmar las identidades locales y a fomentar las relaciones interétnicas. Resta asimismo establecer el papel desempeñado por la persecución religiosa que tuvo lugar a lo largo de esos años; es decir, si contribuyó a incentivar o a entorpecer el desarrollo de las escuelas rurales federales.

Aunque terminó siendo clausurada, la experiencia de la escuela federal en la localidad de Aguacatenango ilustra algunas alianzas y conflictos de intereses despertados por la implementación del proyecto de nación posrevolucionario. En esta oportunidad tenemos indicios que nos conducen a pensar que las autoridades indígenas locales manipularon al maestro y a la comunidad en beneficio propio.

El experimento escolar en Tzimol es el que más se acerca a la tesis de que en las poblaciones rurales más incorporadas a la sociedad nacional el proyecto de la SEP consiguió mejores resultados. Aun así, una escuela que fomentó la unión y la previsión y obtuvo empatía a partir de actividades culturales no logró sustraerse a las consecuencias acarreadas por el cambio de maestros y de directrices políticas nacionales.

El último caso que tratamos aquí presenta la distancia que pudo haber existido entre el éxito en la transmisión del discurso reformista revolucionario y 
las posibilidades de materializarlo. Los reclamos de mayor compromiso social esgrimidos por la Junta de Educación de Socoltenango muestran el conocimiento y uso del discurso educativo federal por parte de los padres de familia. Intuimos que, a finales de los años treinta, en esta localidad el PRM entró con tal fuerza que logró hermanar las actividades escolares a la lucha agraria y a otras reivindicaciones sociales.

En síntesis, con el análisis de los casos aquí presentados pretendemos haber incentivado la investigación acerca de las alianzas y conflictos de intereses que surgieron entre las comunidades, los maestros y las autoridades locales, regionales y federales a raíz de la implementación de los proyectos de la SEP en los campos mexicanos. Asimismo, esperamos haber despertado la curiosidad sobre las formas en que dicho proyecto fue apropiado por diferentes actores con motivaciones particulares. En pocas palabras, quedamos satisfechos si hemos dejado al lector con la sensación de que el proyecto de las escuelas rurales federales no puede comprenderse en términos de éxito o fracaso. Es en sus adaptaciones a condiciones locales donde todavía tenemos mucho por conocer.

\section{Notas}

${ }^{1}$ Adoptamos el término ladino para referirnos a los no indios o mestizos (Bolaños, González y Pérez, 1996; Gould, 1998; Obara-Saeki, 2010).

2 AHSEP, DECI, caja 25, exp. 3, Informe sobre los trabajos desarrollados en los pueblos La Independencia, Las Rosas y Socoltenango y la ranchería de Yoc Na Jab, 1923-1924, f. 145.

3 Dicha afirmación nos parece exagerada. De acuerdo con el censo de 1930, el municipio de Villa Las Rosas tenía un total de 5586 pobladores, de los cuales 1892 eran hablantes de lenguas indígenas, es decir, el 33.9\% (Dirección General de Estadística, 1943).

${ }^{4}$ AHSEP, DER, ERF, caja 12, exp. 29, Escuela rural federal Valentín Gómez Farías, Teopisca, Chis., 1926-1934, f. $\mathrm{s} / \mathrm{n}$. Hasta que se indique lo contrario, las siguientes referencias pertenecen al mismo expediente.

${ }^{5}$ Muy seguramente, en este caso el inspector se refería únicamente al pueblo de Teopisca. Sabemos que el municipio en su totalidad -incluyendo también Amatenango del Valle - llegaba a 5432 habitantes (Dirección General de Estadística, 1935: 11-12).

6 AHSEP, DER, ERF, caja 12, exp. 29, Escuela rural federal Valentín Gómez Farías, Teopisca, Chis., 19261934, El Inspector de la Tercera Zona Escolar, Ramón Campillo, "Opina sobre si la escuela de Teopisca, Las Casas, Chiapas, debe convertirse en primera elemental", Chiapa de Corzo, 17 de abril de 1929, f. s/n.

7 AHSEP, DER, ERF, caja 12, exp. 29, Escuela rural federal Valentín Gómez Farías, Teopisca, Chis., 19261934, El Inspector de la Tercera Zona Escolar, Ramón Campillo, "Opina sobre si la escuela de Teopisca, Las Casas, Chiapas, debe convertirse en primera elemental", Chiapa de Corzo, 17 de Abril de 1929, f. s/n.

${ }^{8}$ AHSEP, DER, ERF, caja 12, exp. 29, Escuela rural federal Valentín Gómez Farías, Teopisca, Chis., 1926-1934, El Director de Educación, Erasto Valle, "Suplicándole ordenar al C. Jefe de Hacienda de San Cristóbal no siga exigiendo la desocupación de los anexos del templo de San Agustín del pueblo de Teopisca, Las Casas, Chis. porque los necesita la Escuela y no tiene otros", Tuxtla Gutiérrez, l de diciembre de 1930, f. s/n.

${ }^{9}$ AHSEP, DER, ERF, caja 12, exp. 22, Escuela rural federal Amatenango, Teopisca, Chis., 1926-1934, f. s/n. Hasta que se aclare lo contrario, en adelante las referencias provienen del mismo expediente.

10 AHSEP, DER, ERF, caja 12, exp. 22, Escuela rural federal Amatenango, Teopisca, Chis., 1926-1934, Inspector Institucional, "Amatenango, Municipalidad de Teopisca, Distrito de Las Casas, Escuela Rural Mixta”, San Cristóbal de Las Casas, 1934, f. s/n.

${ }^{11}$ AHSEP, DER, ERF, caja 12, exp. s/n, Escuela rural federal de Aguacatenango, San Cristóbal, Chis., 1926-1933, f. $\mathrm{s} / \mathrm{n}$. Hasta que sea indicado algo diferente, las siguientes referencias corresponden al mismo expediente. 
12 AHSEP, DER, ERF, caja 12, exp. s/n, Escuela rural federal de Aguacatenango, San Cristóbal, Chis., 19261933, Ángel M. Corzo (inspector), "Informe sintético de visitas de inspección, 23 nov. 1926”, Tuxtla Gutiérrez, 21 de diciembre de 1926, f. s/n; Epigmenio de León (inspector), "Informe sintético de visita de inspección, 10 oct. 1930", 11 de octubre de 1930, f. s/n.

${ }^{13}$ AHSEP, DER, ERF, caja 12, exp. s/n, Escuela rural federal de Aguacatenango, San Cristóbal, Chis., 19261933, Epigmenio de León (inspector), "Informe sintético de visita de inspección, 19 mar. 1930", 22 de marzo de 1930, f. s/n.

${ }^{14}$ AHSEP, DER, ERF, caja 12, exp. s/n, Escuela rural federal de Aguacatenango, San Cristóbal, Chis., 19261933, Agencia Municipal, Aguacatenango, 12 de octubre de 1930, f. s/n; Damián Chávez (maestro rural), "Se remite copia de la inauguración de la Casa de la Escuela", Aguacatenango, Distrito de La Libertad, Chis., 13 de octubre de 1930, f. s/n.

15 AHSEP, DER, ERF, caja 12, exp. s/n, Escuela rural federal de Aguacatenango, San Cristóbal, Chis., 19261933, José Inés Estrada (inspector), “Informe sintético de visita de inspección, 30 abr. -1ํㅡ may. 1931”, 1 de mayo de 1931, f. s/n.

${ }^{16}$ AHSEP, DER, ERF, caja 12, exp. s/n, Escuela rural federal Jaime Nunó, Tzimol, Mun. Tzimol (Ant. Comitán), 1926-1976, f.s/n. Hasta nueva aclaración, los siguientes datos provienen del mismo expediente.

${ }^{17}$ AHSEP, DER, ERF, caja 12, exp. s/n, Escuela rural federal Jaime Nunó, Tzimol, Mun. Tzimol (Ant. Comitán), 1926-1976, "Acta del nuevo Comité de Educación”, ranchería Tzimol, municipio de Comitán, l de agosto de 1929, f. s/n; José Inés Estrada (inspector), "Informe sintético de visitas de inspección, 13 y 14 ene. 1930", 14 de enero de 1930, f. s/n; José Inés Estrada (inspector), "Informe sintético de visitas de inspección, 18 y 19 may. 193l”, 20 de mayo de 1931, f. s/n; José Inés Estrada (inspector), "Informe sintético de visitas de inspección, 20 abr. 1932”, 20 de abril de 1932, f. s/n.
${ }^{18}$ AHSEP, DER, ERF, caja 12, exp. s/n, Escuela rural federal Jaime Nunó, Tzimol, Mun. Tzimol (Ant. Comitán), 1926-1976, Fernando Utrilla (director temporal de la escuela), "Se acusa recibo de Nota de felicitación y se dá cuenta del éxito de un festival en la Esc. Rural 'El Puente', adjuntando fotografías", Tzimol, 15 de agosto de 1937, f. s/n.

${ }^{19}$ AHSEP, DER, ERF, caja 12, exp. s/n, Escuela rural federal Socoltenango, Socoltenango, 1926-1977, f. s/n. En adelante todas las referencias corresponden al mismo expediente.

${ }^{20}$ AHSEP, DER, ERF, caja 12, exp. s/n, Escuela rural federal Socoltenango, Socoltenango, 1926-1977, El Comité de Educación al Director General de Educación, "Suplicando atienda nuestras quejas para que se corrijan las deficiencias de esta Escuela" Socoltenango, 23 de noviembre de 1929, f. s/n.

${ }^{21}$ AHSEP, DER, ERF, caja 12, exp. s/n, Escuela rural federal Socoltenango, Socoltenango, 1926-1977, Los suscritos vecinos del pueblo de Socoltenango al director de Educación Federal, Socoltenango, 5 de octubre de 1938, f. s/n.

\section{Bibliografía}

Arce Gurza, Francisco (1981), “En busca de una educación revolucionaria: 1924-1934”, en Vázquez, Josefina Zoraida et al., Ensayos sobre historiografía de la educación en México, México: El Colegio de México, pp. 145-187.

Bolaños, Ligia, Yamileth González y María Pérez (1996), "El ladino: base del desarrollo cultural hegemónico en Centroamérica", en Identidades y producciones culturales en América Latina, San José: Universidad de Costa Rica, pp. 87-152.

Dirección General de Estadística (1935), Quinto censo de población, 15 de mayo 1930. Estado de Chiapas, México: Secretaría de la Economía Nacional.

Dirección General de Estadística (1943), 6º Censo de población, 1940. Chiapas, México: Secretaría de la Economía Nacional. 
Fell, Claude (1989), José Vasconcelos. Los años del águila, 19201925, México: UNAM.

Gould, Jeffrey L. (1998), To Die in this way. Nicaraguans Indians and the Myth of Mestizaje, 1880-1965, Durham y Londres: Duke University Press.

Heath, Shirley Brice (1972), La politica del lenguaje en México: de la colonia a la nación, México: SEP/Instituto Nacional Indigenista.

Helbig, Karl M. (1964), La cuenca superior del río Grijalva. Un estudio regional de Chiapas, sureste de México, Tuxtla Gutiérrez: Instituto de Ciencias y Artes de Chiapas.

Lewis, Stephen (2005), The Ambivalent Revolution. Forging State and Nation in Chiapas, 1910-1945, Albuquerque: University of New Mexico Press.

Lewis, Stephen (2006), "The Nation, Education, and the 'Indian Problem' in Mexico, 1920-1940", en Vaughan, Mary Kay y Stephen Lewis (eds.), The Eagle and the Virgin. Nation and Cultural Revolution in Mexico, 1920-1940, Durham y Londres: Duke University press, pp. 176-195.

Loyo, Engracia (1996), "La empresa redentora. La Casa del Estudiante Indígena", en Historia Mexicana, vol. XLVI, núm. 1, pp. 99-131.

Obara-Saeki, Tadashi (2010), Ladinización sin mestizaje. Historia demográfica del área chiapaneca 1748-1813, Tuxtla Gutiérrez: Consejo Estatal para las Culturas y las Artes de Chiapas.

Quintanilla, Susana y Mary Kay Vaughan (1997), "Presentación", en Quintanilla, Susana y Mary Kay Vaughan (comps.),Escuelay sociedadenelperiodocardenista, México: Fondo de Cultura Económica, pp. 7-43.
Sáenz, Moisés (1936), Carapan: bosquejo de una experiencia, Lima: Editorial Gil.

Urías Horcasitas, Beatriz (2002), "Las ciencias sociales en la encrucijada del poder: Manuel Gamio (19201940)", en Revista Mexicana de Sociología, vol. 64, núm. 3, pp. 93-121.

Urías Horcasitas, Beatriz (2007), Historias secretas del racismo en México (1920-1950), México: Tusquets.

Vaughan, Mary Kay (2000), La política cultural en la Revolución. Maestros, campesinos y escuelas en México, 19301940, México: Fondo de Cultura Económica.

Viqueira, Juan Pedro (1995), "Chiapas y sus regiones", en Viqueira, Juan Pedro y Mario Humberto Ruz (eds.), Chiapas: los rumbos de otra historia, México: Universidad Nacional Autónoma de México/ Centro de Investigaciones y Estudios Superiores en Antropología Social, pp. 19-40.

Viqueira, Juan Pedro (1997), Cronotopología de una región rebelde. La construcción histórica de los espacios sociales en la alcaldía mayor de Chiapas (1520-1720), (tesis de doctorado), Escuela de Altos Estudios en Ciencias Sociales, París, Francia.

\section{Fuentes de archivo}

AHSEP, DECI: Archivo Histórico de la Secretaría de Educación Pública, Departamento de Educación y Cultura Indígena, México, D.F.

AHSEP, DER, ERF: Archivo Histórico de la Secretaría de Educación Pública, Departamento de Escuelas Rurales, Escuelas Rurales Federales, México, D.F. 
Mapa 1. La región de San Bartolomé de Los Llanos

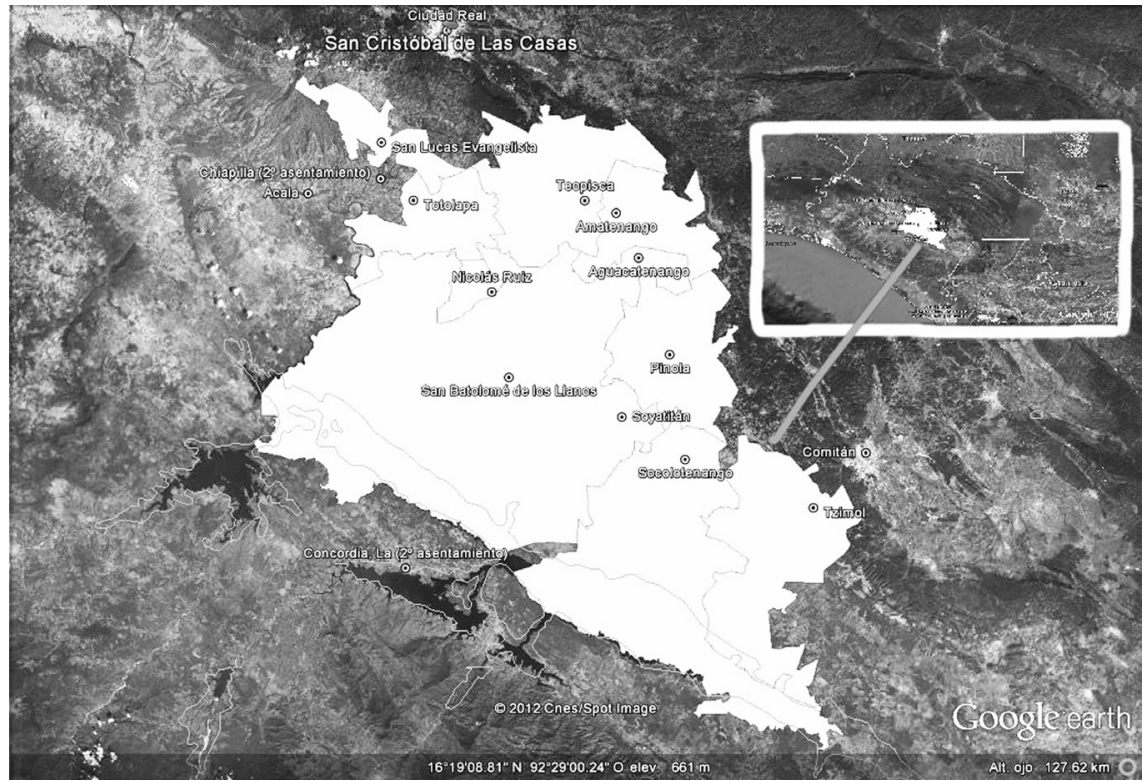

Fuente: Google Earth con base en Helbig (1964: 27) y Viqueira (1995: 22).

Foto 1. Teatro de títeres de la Escuela Rural Federal Tzimol, Comitán, Chiapas. Maestro Fernando Utrilla, circa 1937.

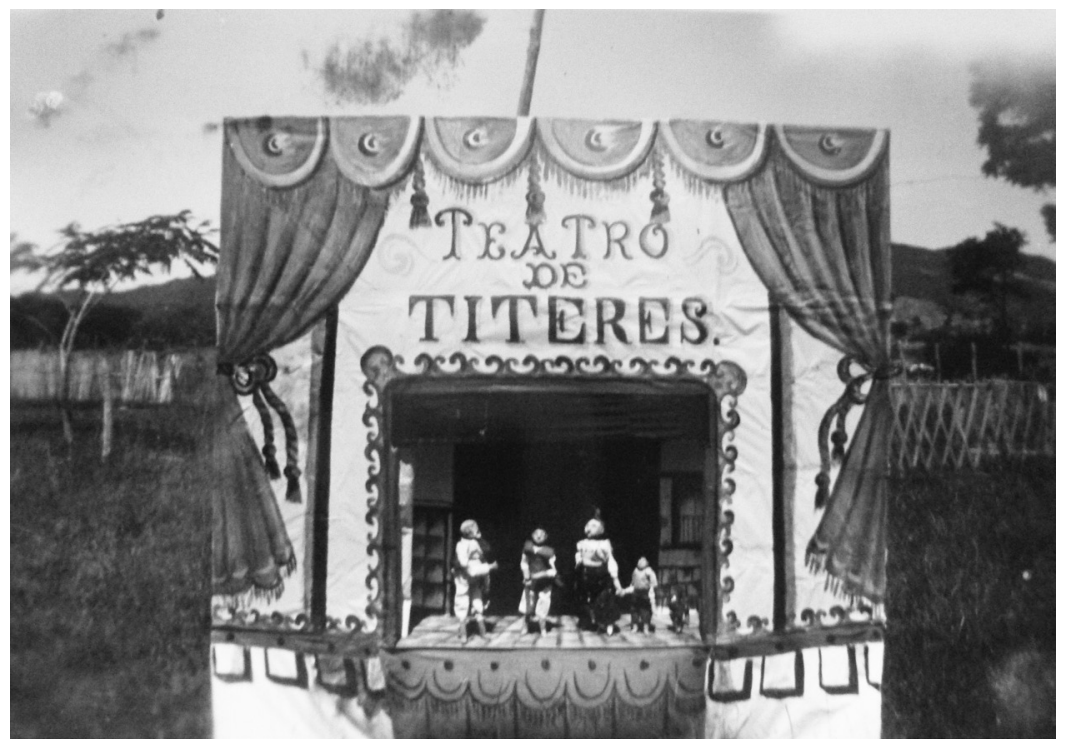

Fuente: AHSEP, DER, ERF, caja 12, exp. S/N, Escuela rural federal Jaime Nuno, Tzimol, Mun. Tzimol (Ant. Comitán), 1926-1976. 